\title{
Spiral magnetism, spin flop, and pressure-induced ferromagnetism in the negative charge-transfer-gap insulator $\mathrm{Sr}_{2} \mathrm{FeO}_{4}$
}

\author{
Peter Adler $\odot,{ }^{1, *}$ Manfred Reehuis, ${ }^{2}$ Norbert Stüßer, ${ }^{2}$ Sergey A. Medvedev,${ }^{1}$ Michael Nicklas $\odot,{ }^{1}$ Darren C. Peets $\odot,{ }^{3}$ \\ Joel Bertinshaw, ${ }^{4}$ Christian Kolle Christensen, ${ }^{5}$ Martin Etter $\odot,{ }^{5}$ Andreas Hoser $\odot,{ }^{2}$ Liane Schröder, ${ }^{1}$ Patrick Merz, ${ }^{1}$ \\ Walter Schnelle, ${ }^{1}$ Armin Schulz $\odot,{ }^{5}$ Qingge Mu, ${ }^{1}$ Dimitrios Bessas $\odot,{ }^{6}$ Aleksandr Chumakov $\odot,{ }^{6}$ \\ Martin Jansen $\odot,{ }^{1,4, \dagger}$ and Claudia Felser ${ }^{1}$ \\ ${ }^{1}$ Max-Planck-Institut für Chemische Physik fester Stoffe, 01187 Dresden, Germany \\ ${ }^{2}$ Helmholtz-Zentrum für Materialien und Energie, 14109 Berlin, Germany \\ ${ }^{3}$ Institut für Festkörper- und Materialphysik, Technische Universität Dresden, 01069 Dresden, Germany \\ ${ }^{4}$ Max-Planck-Institut für Festkörperforschung, 70569 Stuttgart, Germany \\ ${ }^{5}$ Deutsches Elektronen-Synchrotron (DESY), 22607 Hamburg, Germany \\ ${ }^{6}$ ESRF-The European Synchrotron, CS 40220, 38043 Grenoble Cedex 9, France
}

(Received 1 September 2021; revised 3 February 2022; accepted 3 February 2022; published 17 February 2022)

\begin{abstract}
Iron(IV) oxides are strongly correlated materials with negative charge-transfer energy (negative $\Delta$ ), and exhibit peculiar electronic and magnetic properties such as topological helical spin structures in the metallic cubic perovskite $\mathrm{SrFeO}_{3}$. Here, the spin structure of the layered negative- $\Delta$ insulator $\mathrm{Sr}_{2} \mathrm{FeO}_{4}$ was studied by powder neutron diffraction in zero field and magnetic fields up to $6.5 \mathrm{~T}$. Below $T_{\mathrm{N}}=56 \mathrm{~K}, \mathrm{Sr}_{2} \mathrm{FeO}_{4}$ adopts an elliptical cycloidal spin structure with modulated magnetic moments between 1.9 and $3.5 \mu_{\mathrm{B}}$ and a propagation vector $\boldsymbol{k}=(\tau, \tau, 0)$ with $\tau=0.137$. With increasing magnetic field the spin structure undergoes a spin-flop transition near $5 \mathrm{~T}$. Synchrotron ${ }^{57} \mathrm{Fe}$-Mössbauer spectroscopy reveals that the spin spiral transforms to a ferromagnetic structure at pressures between 5 and $8 \mathrm{GPa}$, just in the pressure range where a Raman-active phonon nonintrinsic to the $\mathrm{K}_{2} \mathrm{NiF}_{4}$-type crystal structure vanishes. These results indicate an insulating ground state which is stabilized by a hidden structural distortion and differs from the charge disproportionation in other Fe(IV) oxides.
\end{abstract}

DOI: 10.1103/PhysRevB.105.054417

\section{INTRODUCTION}

The interplay between charge, orbital, spin, and lattice degrees of freedom in compounds with strong electron correlations leads to a large, complex array of structural and physical properties [1]. Particularly interesting is the crossover regime between localized and itinerant electronic behavior where the correlation energy $U$ and the electronic bandwidth $W$ are comparable in magnitude. Such an electronic situation seems to occur in the iron-based superconductors and their magnetic relatives [2]. A crossover between localized and itinerant electronic behavior can also be realized in strongly covalent high-oxidation state transition metal oxides, which in terms of a generalized Zaanen-Sawatzky-Allen diagram [3] can be described as negative charge-transfer energy (negative- $\Delta$ ) materials. Owing to the negative $\Delta$, both insulating as well as metallic states are dominated by holes

\footnotetext{
*adler@cpfs.mpg.de

${ }^{\dagger}$ M.Jansen@fkf.mpg.de
}

Published by the American Physical Society under the terms of the Creative Commons Attribution 4.0 International license. Further distribution of this work must maintain attribution to the author(s) and the published article's title, journal citation, and DOI. Open access publication funded by the Max Planck Society. in the oxygen bands and thus, in the insulating members a $p-p$ type energy gap occurs. Examples of negative- $\Delta$ oxides include $\mathrm{NaCuO}_{2}[4]$ and several rare-earth nickelates $L n \mathrm{NiO}_{3}$ the insulating ground states of which feature a charge disproportion (CD) transition of the nominal $\mathrm{Ni}^{3+}$ ions accompanied by a breathing-type structural distortion $[5,6]$. The nature of this CD is under debate, for instance it has been considered as site-selective Mott transition [7], charge-ordering without charge transfer [8] or as bond disproportionation [9]. It has been suggested that the $\mathrm{CD}$ occurs in the crossover region between localized and itinerant electron behavior as an alternative to the Jahn-Teller effect [10] which is expected in the localized-electron limit for the $t_{2 g}{ }^{6} e_{g}{ }^{1}$ electron configuration. The CD in such nickelates has been described as $2 d^{8} L^{-1}$ $\left(t_{2 g}{ }^{6} e_{g}{ }^{1}\right) \rightarrow d^{8}\left(t_{2 g}{ }^{6} e_{g}{ }^{2}\right)+d^{8} L^{-2}\left(t_{2 g}{ }^{6}\right)$ where $L^{-n}$ denotes holes in the oxygen bands [6,11]. An analog, however more complex charge ordering pattern was reported for the hexagonal antiferromagnet $\mathrm{AgNiO}_{2}[12,13]$.

The insulating states of several $\mathrm{Fe}^{4+}\left(3 d^{4}\right)$ ferrates like $\mathrm{CaFeO}_{3}$ [14,15] or $\mathrm{Sr}_{3} \mathrm{Fe}_{2} \mathrm{O}_{7}$ [16-19] are known to exhibit a comparable $\mathrm{CD}$, whereas metallic $\mathrm{SrFeO}_{3}[20,21]$ as well as the cubic phase of $\mathrm{BaFeO}_{3}$ [22] do not show any $\mathrm{CD}$. These ferrates were shown to also be negative- $\Delta$ materials [23] and the CD state was formulated as $2 d^{5} L^{-1}$ $\left(t_{2 g}{ }^{3} e_{g}{ }^{1}\right) \rightarrow d^{5}\left(t_{2 g}{ }^{3} e_{g}{ }^{2}\right)+d^{5} L^{-2}\left(t_{2 g}{ }^{3} e_{g}{ }^{0}\right)$, where, similar to the nickelates, singly occupied $\sigma$-type $e_{g} *$ orbitals occur, being however additionally coupled to more-localized $\pi$-type $t_{2 g}$ 
electrons. Many of these ferrates exhibit helical spin structures [15,22,24,25], which have been explained in terms of competing ferro- and antiferromagnetic interactions [24,25] or by a double-exchange mechanism where in the negative- $\Delta$ regime helical ordering may naturally emerge without additional superexchange interactions [26]. Magnetotransport studies on $\mathrm{SrFeO}_{3}[27,28]$ indicated a variety of distinct helical phases, some of which exhibit unconventional Hall effects [29]. Recently it was demonstrated that $\mathrm{SrFeO}_{3}$ adopts helical spin structures with multiple propagation vectors [30] and hosts topological spin textures despite the absence of Dzyaloshinskii-Moriya interactions in this centrosymmetric cubic perovskite.

Less explored than the $n=\infty\left(\mathrm{SrFeO}_{3}\right)$ and $n=$ $2\left(\mathrm{Sr}_{3} \mathrm{Fe}_{2} \mathrm{O}_{7}\right)$ members of the Ruddlesden-Popper series $\mathrm{Sr}_{n+1} \mathrm{Fe}_{n} \mathrm{O}_{3 n+1}$ is the $n=1$ member $\mathrm{Sr}_{2} \mathrm{FeO}_{4}$, an insulator with a $\mathrm{K}_{2} \mathrm{NiF}_{4}$-type crystal structure [31,32]. Complex Mössbauer spectra indicated a helical spin structure here too [16], but the detailed spin arrangement has remained elusive. $\mathrm{Sr}_{2} \mathrm{FeO}_{4}$ does not exhibit a CD of $\mathrm{Fe}^{4+}$, but a Raman band nonintrinsic to the $I 4 / \mathrm{mmm}$ structure which is suppressed at pressures near $6 \mathrm{GPa}$ [33] may indicate another type of electronic instability. Metallization of $\mathrm{Sr}_{2} \mathrm{FeO}_{4}$ at $18 \mathrm{GPa}$ and a change in spin structure have been assigned to the closure of the $p-p$ type gap in this negative- $\Delta$ layered ferrate [34]. In this work we report a powder neutron diffraction study which reveals that $\mathrm{Sr}_{2} \mathrm{FeO}_{4}$ forms an elliptical cycloidal spin spiral undergoing a spin-flop transition in a magnetic field of about $5 \mathrm{~T}$. Using ${ }^{57} \mathrm{Fe}$ synchrotron Mössbauer spectroscopy it is shown that the spin spiral transforms to a ferromagnetic spin alignment at pressures between 5 and $8 \mathrm{GPa}$. Our results shed new light on the nature of insulating states and emerging spin structures in negative- $\Delta$ materials which may also be of relevance for understanding topological spin textures in this class of compounds.

\section{EXPERIMENTAL DETAILS}

$\mathrm{Sr}_{2} \mathrm{FeO}_{4}$ was synthesized from $\mathrm{SrO}$ and $\mathrm{Fe}_{2} \mathrm{O}_{3}$ in an autoclave at a temperature of $500^{\circ} \mathrm{C}$ and an oxygen pressure of $2.5 \mathrm{kbar}$ following the method described in Ref. [35]. The sample was confirmed to be single-phase by laboratory powder x-ray diffraction. All sample batches used within this study were characterized by ${ }^{57} \mathrm{Fe}$ Mössbauer spectroscopy. Residual $\mathrm{Fe}^{3+}$ fractions between 1 and 3\% suggest the composition $\mathrm{Sr}_{2} \mathrm{FeO}_{3.99(1)}$.

Powder neutron diffraction experiments on $\mathrm{Sr}_{2} \mathrm{FeO}_{4}$ were carried out on the instruments E2 [36] and E6 [37] at the BER II reactor of the Helmholtz-Zentrum Berlin. Both instruments use a pyrolytic graphite monochromator selecting the neutron wavelengths $\lambda=2.380$ (E2) and $\lambda=2.417 \AA$ (E6), respectively. Powder patterns were collected between the following diffraction angles: 3.7 and $79.4^{\circ}$ (E2); 4.9 and $136.6^{\circ}$ (E6). The powder sample was placed in a cylindrical vanadium container with the dimensions $d=6 \mathrm{~mm}$ and $h=5 \mathrm{~cm}$. To investigate in detail the magnetic structure of $\mathrm{Sr}_{2} \mathrm{FeO}_{4}$ we collected diffraction patterns at 1.8 and $80 \mathrm{~K}$ on E2 well below and above the magnetic ordering temperature $T_{\mathrm{N}}=56 \mathrm{~K}$. Here we measured with higher counting statistics and a better instrumental resolution using a $15^{\prime}$ collimator. The change of the structural and magnetic parameters as a function of temperature was investigated on the instrument E6 between 1.8 and $62 \mathrm{~K}$. Furthermore, on the instrument E2 the change of the magnetic structure was investigated at $1.8 \mathrm{~K}$ as a function of a vertical magnetic field up to $6.5 \mathrm{~T}$. Rietveld refinements were carried out with the program FullProf [38] using the nuclear scattering lengths $b(\mathrm{O})=5.805 \mathrm{fm}, b(\mathrm{Fe})=9.54 \mathrm{fm}$, and $b(\mathrm{Sr})=7.02 \mathrm{fm}[39]$. The magnetic form factors of the $\mathrm{Fe}$ atoms were taken from Ref. [39].

The crystal structure of $\mathrm{Sr}_{2} \mathrm{FeO}_{4}$ has been investigated by synchrotron powder $\mathrm{x}$-ray diffraction at 100,300 , and $500 \mathrm{~K}$ using the wavelength $\lambda=0.20761 \AA$ at the beamline P02.1 of the PETRA III x-ray radiation source at Deutsches Elektronensynchrotron DESY, Hamburg. The powdered sample was spun during the measurement and the temperature was controlled by an Oxford-Cryostream 700. Data collection was performed on a Varex-XRD $4343 \mathrm{CT}$ area detector $(150 \times$ $150 \mu \mathrm{m}^{2}$ pixel size, $2880 \times 2880$ pixel area) at a sample to detector distance of approximately $2200 \mathrm{~mm}$. Two-dimensional data was subsequently integrated to one-dimensional diffraction patterns using the DAWN software package.

Mössbauer spectra of $\mathrm{Sr}_{2} \mathrm{FeO}_{4}$ were collected between 5 and $290 \mathrm{~K}$ at ambient pressure with a conventional WISSEL Mössbauer spectrometer which was equipped with a ${ }^{57} \mathrm{Co} / \mathrm{Rh}$ radioactive source and operated in constant acceleration mode. The spectra were collected on a sample with $20 \%{ }^{57} \mathrm{Fe}$ enrichment which also was used for high-pressure studies at $8 \mathrm{GPa}$. A few milligrams of the sample were mixed with boron nitride and distributed in a plexiglass container which was placed inside a JANIS SHI-850-5 closed-cycle refrigerator.

High-pressure Mössbauer spectra at 5.0 and $8.1 \mathrm{GPa}$ at $3 \mathrm{~K}$ were recorded in diamond anvil cells with the samples deposited in the pre-indented holes of a tungsten gasket, using the synchrotron Mössbauer source (SMS) [40] at beamline ID18 of ESRF [41], Grenoble. Silicone oil was used as pressure-transmitting medium. The pressure was determined by the ruby luminescence method. The sample used for the 5 -GPa experiment was enriched to $10 \%{ }^{57} \mathrm{Fe}$. At $8 \mathrm{GPa}$ in addition to the zero-field spectrum a SMS spectrum in a longitudinal magnetic field of $2 \mathrm{~T}$ was measured. The spectra were evaluated with the program MOSSWINN [42] within the thin-absorber approximation in case of the ambient-pressure spectra, and with the transmission integral and a squared Lorentzian for the source function in case of the high-pressure SMS spectra.

Temperature-dependent and isothermal dc magnetization measurements were performed with a MPMS3 magnetometer [Quantum Design, using superconducting quantum interference device (SQUID) signal detection, data in Fig. 1(a) labeled with "S"] in magnetic fields up to $7 \mathrm{~T}$ and with a field-extraction technique using the ACMS option in a PPMS (Quantum Design, data in Fig. 1(a) labeled with "P") up to $14 \mathrm{~T}$. Ac susceptibility data were taken with the same equipment option of the PPMS.

Heat capacity measurements with a relaxation method were performed on a cold-pressed pellet using the HC option of a PPMS in zero field.

Raman spectra were obtained between 5 and $300 \mathrm{~K}$ with a Jobin-Yvon-LabRAM-V010 single grating spectrometer, 
(a)

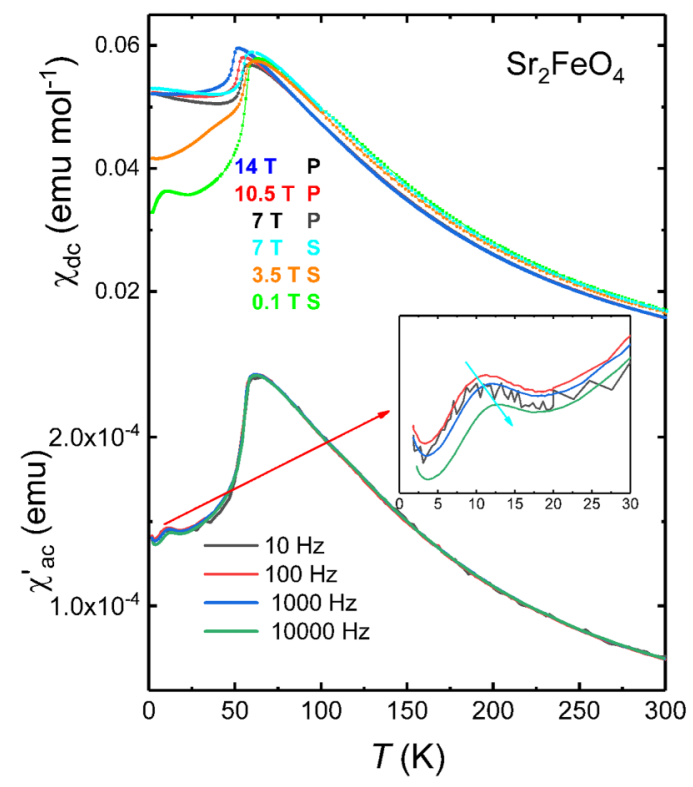

(b)

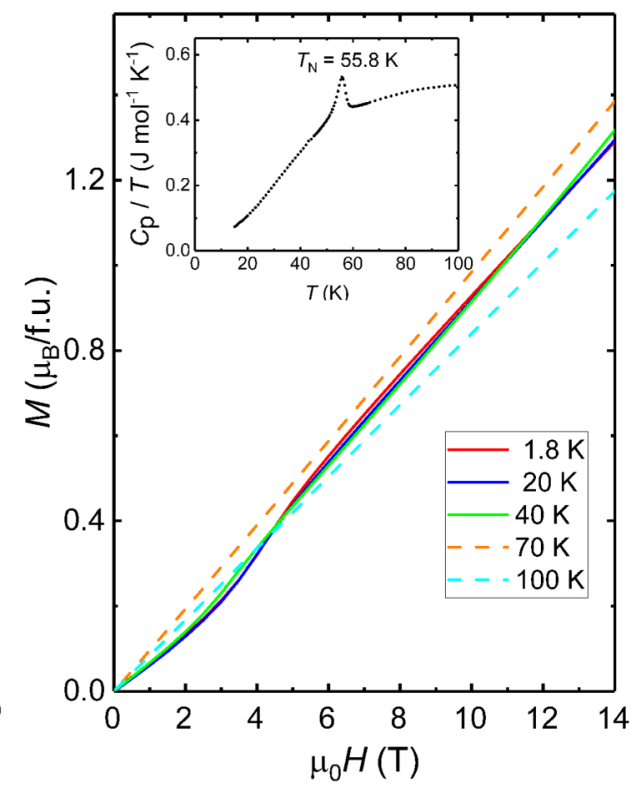

FIG. 1. Magnetic properties of $\mathrm{Sr}_{2} \mathrm{FeO}_{4}$. (a) Top: dc magnetic susceptibility measured after zero-field cooling in different magnetic fields. $\mathrm{S}$ and $\mathrm{P}$ correspond to measurements using a SQUID and a PPMS-based magnetometer, respectively. Bottom: Real part of the ac susceptibility measured in zero field at the indicated frequencies; the inset highlights a weak feature near $10 \mathrm{~K}$. (b) Isothermal magnetization measured at the indicated temperatures. Inset: Heat capacity $C_{p} / T$ in zero field as a function of temperature.

equipped with a double super-razor-edge filter and a Peltier cooled CCD camera, in quasi-backscattering geometry using the linearly polarized $632.8 \mathrm{~nm}$ line of a $\mathrm{He} / \mathrm{Ne}$ gas laser. The sample was placed in a micro-cryo-type cryostat from Cryovac, in a holder with copper cold finger with a vacuum better than $1 \times 10^{-6}$ mbar. To avoid sample decomposition under irradiation a small laser power of $0.2 \mathrm{~W}$ was used, the spot size was $10 \mu \mathrm{m}$. Measurements were taken at temperatures ranging between 5 and $300 \mathrm{~K}$ with $0.5 \mathrm{~K}$ accuracy.

\section{RESULTS AND DISCUSSION}

\section{A. Magnetic properties}

The temperature dependence of the dc magnetic susceptibility $\chi=M / H$, where $M$ is the magnetization and $H$ the applied magnetic field, shows for $\mu_{0} H=0.1 \mathrm{~T}$ a broad maximum near $60 \mathrm{~K}$ [Fig. 1(a), top], indicating an antiferromagnetic ordering, in agreement with previous works [16,32]. Similarly, a frequency-independent maximum is apparent in the real part of the ac susceptibility curves [Fig. 1(a), bottom]. From the $\lambda$-type anomaly in the specific heat $\left(C_{\mathrm{p}}\right)$ data [inset of Fig. 1(b)] a Néel temperature $T_{\mathrm{N}}$ of 55.8(2) K was determined. A weak feature near $10 \mathrm{~K}$, which is visible in both zero-field cooled dc and ac susceptibility data and which shows a slight frequency dependence in the ac data [inset of Fig. 1(a)] is possibly related to the presence of oxygen defects. Remarkably, the susceptibility curves show a distinct dependence on the applied field for temperatures below the susceptibility maximum. Furthermore, the maximum shifts to lower temperatures. From the cusps in Fisher's heat capacity $\mathrm{d}(\chi T) / \mathrm{d} T$ data it is estimated that $T_{\mathrm{N}}$ decreases by $8 \mathrm{~K}$ from $\mu_{0} H=0.1$ to $14 \mathrm{~T}$. Isothermal $M(H)$ curves were measured between -14 and $+14 \mathrm{~T}$ at selected temperatures between 2 and $100 \mathrm{~K}$ [see Fig. 1(b) and Ref. [43]]. While the $M(H)$ curves at 70 and $100 \mathrm{~K}$ are linear, in agreement with paramagnetic behavior, smooth deviations from linearity between 3 and $6 \mathrm{~T}$ below $T_{\mathrm{N}}$ indicate a broadened spin-flop (SF) transition in the polycrystalline sample [44].

\section{B. Magnetic structure}

To unravel the magnetic structure of $\mathrm{Sr}_{2} \mathrm{FeO}_{4}$ we have collected powder neutron diffraction data on the instrument E2 in the magnetically ordered and paramagnetic phase at 1.8 and $80 \mathrm{~K}$, respectively (Fig. 2). In agreement with Ref. [16], the crystal structure at $80 \mathrm{~K}$ is well refined in the tetragonal space group $I 4 / \mathrm{mmm}$. In the pattern collected at $1.8 \mathrm{~K}$ several additional magnetic reflections are observable, where the first and strongest magnetic reflection, observed at $2 \theta=6.9^{\circ}$, could be indexed as $(0.13660 .13660)_{\mathrm{M}}$ (Fig. 2). This indicates that the propagation vector is $\boldsymbol{k}=(\tau, \tau, 0)$, where the incommensurate magnetic reflections can be generated with the rule $(h k l)_{\mathrm{M}}=(h k l)_{\mathrm{N}} \pm \boldsymbol{k}$. The magnetic reflections are better obtained after subtracting the nuclear pattern as shown in Fig. 3(a). Initially, we have tried to determine the spin structure by carrying out Rietveld refinements considering different models. Here, it became apparent that some reflections reveal peak broadenings caused either by slightly superimposed diffuse scattering or by strain effects. Further, some magnetic peaks overlap with nuclear ones and the background is partially irregular in a few angular regions. Therefore, for the refinements of the magnetic structure integrated intensities (given in $F_{\mathrm{M}}{ }^{2}$ ) of eight independent magnetic reflections were determined from multiple-Gaussian fits of the pattern at $1.8 \mathrm{~K}$ (Table I). Structure factors of nuclear reflections were taken to 


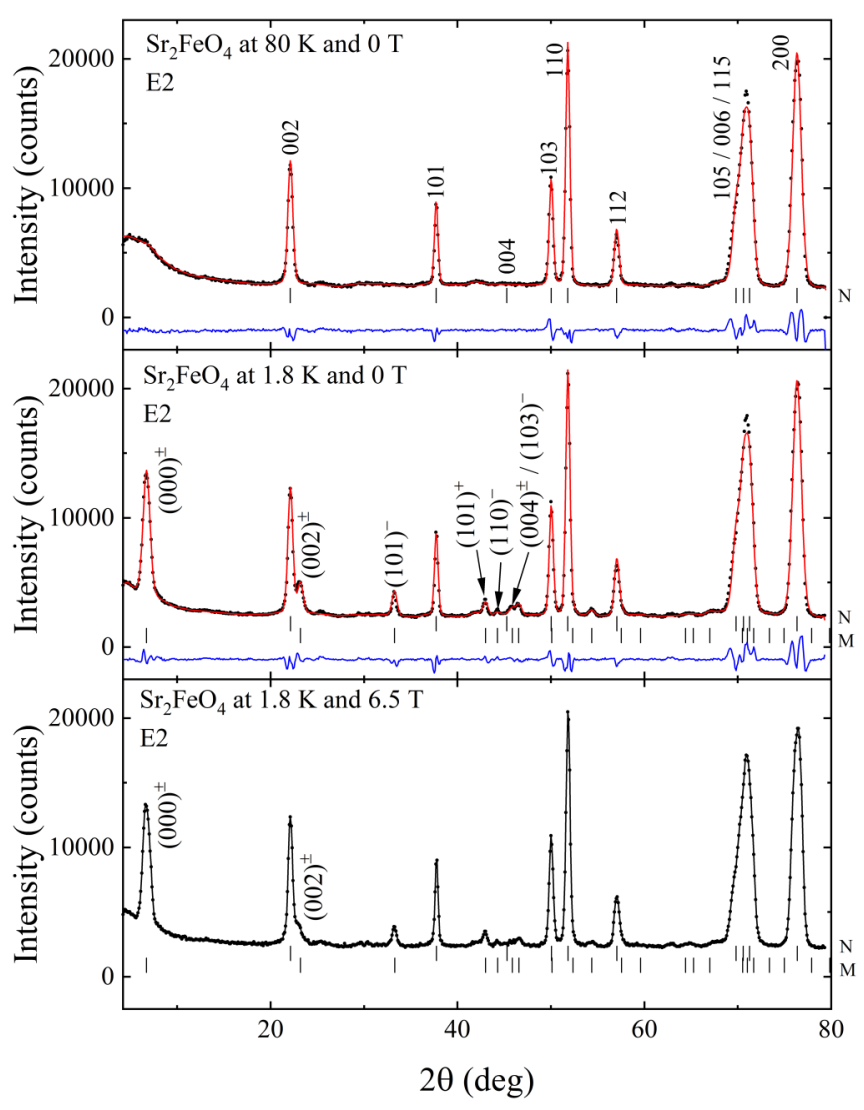

FIG. 2. Powder neutron diffraction patterns of $\mathrm{Sr}_{2} \mathrm{FeO}_{4}$. Data were collected on diffractometer E2 $(\lambda=2.380 \AA)$ at 80 and $1.8 \mathrm{~K}$ (top and middle panel) at zero field. The observed magnetic reflections at $1.8 \mathrm{~K}$ are generated with the rule $(h k l)_{\mathrm{M}}=(h k l)_{\mathrm{N}} \pm \boldsymbol{k}$, where the incommensurate propagation vector is found to be $\boldsymbol{k}=$ $(\tau, \tau, 0)$ with $\tau=0.1366(2)$. In an applied vertical magnetic field of 6.5 T magnetic satellites with higher $l$ like $(002)^{ \pm},(004)^{ \pm},(103)^{-}$ and $(103)^{+}$show a decrease in intensity (bottom panel). The results from Rietveld refinements are only shown for the data sets collected in zero field, where for the magnetic structure at $1.8 \mathrm{~K}$ we used Model II. The calculated patterns (red solid line) are compared with the observations (black-filled circles). The positions of the generated magnetic reflections (black bars) as well as the difference patterns $\left(I_{\mathrm{obs}}-I_{\text {cal }}\right)$ (blue solid line) are also shown. In the case of the data set collected at $6.5 \mathrm{~T}$ only the observed intensities are shown and the solid black line is a guide-to-the-eye.

determine the overall scale factor to calculate the magnitude of the magnetic moments.

The best agreement between experimental and calculated data is obtained for the model of an elliptical cycloidal spiral that is confined to the tetragonal $a b$ plane (labeled as Model I) and described by an incommensurate wave vector $\boldsymbol{k}=$ $(0.137,0.137,0)$, with a minor moment $\mu_{\min }$ of $1.9(2) \mu_{\mathrm{B}}$ and a major moment $\boldsymbol{\mu}_{\text {maj }}$ of 3.46(5) $\mu_{\mathrm{B}}$. In tetragonal symmetry, one has to consider two symmetrically equivalent propagation vectors $(\tau, \tau, 0)$ and $(\tau,-\tau, 0)$ and, independently, two domains arise from two symmetry-related spin directions. The data analysis shows that a successful refinement was obtained for a model in which only one type of domain is present with the major moment $\boldsymbol{\mu}_{\text {maj }}$ perpendicular to the wave vectors. Elliptical spin spirals allowing for a $z$ component either on the minor or on the major axis (labeled as Model II and Model III) also yield satisfying fits of similar quality; a small moment of $0.9 \pm 0.5 \mu_{\mathrm{B}}$ is refined indicating that the spin ellipse tilts only weakly out of the tetragonal plane with an angle of 27(16) and $14(11)^{\circ}$ obtained for models II and III, respectively. Further details of the refinements are given in Table I. If a circular spiral is assumed for Model II (labeled here as Model IIc) which is also allowed to turn out of the $a b$ plane the residual increases from 0.055 to 0.094 . This results in a change of the tilting angle from $27(16)$ to $43(7)^{\circ}$. In contrast, the use of a circular spiral for Model III does not lead to a definite solution. An elliptical modulation of the magnetic moments is also supported by the Mössbauer spectra as discussed in Sec. E.

We also considered the possibility of a circular helical spin structure, where both moments $\mu_{a b}$ and $\mu_{c}$ are perpendicular to $\boldsymbol{k}$. The refinements resulted in a poor residual $R_{\mathrm{M}}=0.17$. Here it was seen that $F_{\mathrm{M} \text {,obs }}{ }^{2}$ and $F_{\mathrm{M} \text {,cal }}{ }^{2}$ of the reflection $(\tau \tau 0)_{\mathrm{M}}$ show a good agreement, but ${F_{\mathrm{M}, \text { cal }}}^{2}$ of the $(\tau \tau 2)_{\mathrm{M}}$ reflections was found to be too small. In the case of an elliptical helical spin structure a better residual $R_{\mathrm{M}}=0.11$ is obtained which is, however, considerably larger than those obtained for the models given above. This leads to a smaller moment $\mu_{c}=1.8(7) \mu_{\mathrm{B}}$ and a larger moment $\mu_{a b}=3.3(7) \mu_{\mathrm{B}}$.

From temperature-dependent measurements $T_{\mathrm{N}}=56(2) \mathrm{K}$ was obtained [Fig. 3(b)], in good agreement with the $C_{\mathrm{p}}$ results. The spin structure of Model II is illustrated in Fig. 3(d) and the results from refinements assuming different models for the spin structure are summarized in Table I. It is evident that the moments are essentially oriented within the $a b$ plane but the present powder neutron data do not allow to decide unambiguously whether or not there is a certain out-of-plane tilting.

An elliptical helical spin structure with $\boldsymbol{k}=(\tau, \tau, 1)$ and a similar $\tau=0.142$ was found for the related RuddlesdenPopper series member $\mathrm{Sr}_{3} \mathrm{Fe}_{2} \mathrm{O}_{7}$, but with less-pronounced anisotropy of the moments $\left(\mu_{c} \sim 3.0 \mu_{\mathrm{B}}\right.$ and $\left.\mu_{a, b} \sim 3.5 \mu_{\mathrm{B}}\right)$ [25]. From inelastic neutron scattering experiments competing ferromagnetic nearest neighbor $\left(\mathrm{NN}, J_{1}=7.2 \mathrm{meV}\right)$ and antiferromagnetic next-nearest neighbor (NNN, $J_{2}=$ $-1.05 \mathrm{meV}, J_{3}=-2.1 \mathrm{meV}$ ) in-plane interactions were derived, which-due to the similar $\mathrm{Fe}-\mathrm{O}$ bond distances-are expected to be of comparable size in $\mathrm{Sr}_{2} \mathrm{FeO}_{4}$. In contrast to $\mathrm{Sr}_{3} \mathrm{Fe}_{2} \mathrm{O}_{7}$, the single-layer crystal structure does not give rise to a ferromagnetic $\mathrm{NN}$ interaction along $c$, which rationalizes why the spins in $\mathrm{Sr}_{2} \mathrm{FeO}_{4}$ are oriented near the $a b$ plane.

\section{Spin-flop transition}

The $M(H)$ measurements indicate a spin-flop transition which is induced by an applied magnetic field between 3 and 6 T. In a SF phase all spin ellipses lie inside the SF plane which is perpendicular to the field direction. Neutron diffraction can deliver insight on the field dependent transition as shown in the powder patterns collected at $1.8 \mathrm{~K}$ in vertical fields of 0 and $6.5 \mathrm{~T}$ (Fig. 2). The data reveal that the wave vector of the cycloidal spiral is not affected by the field and visible changes in intensity become related to reorientations of the spin ellipses during the flop. For the analysis of the 
(a)
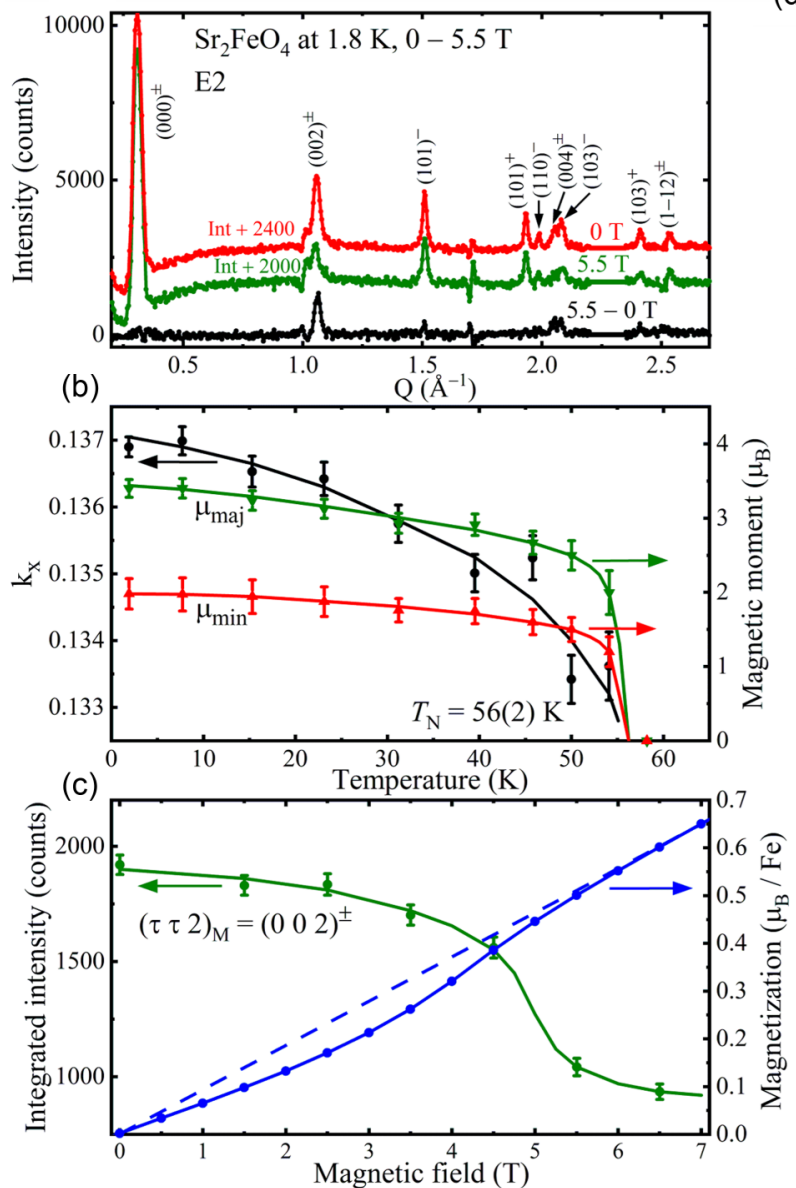

(d)
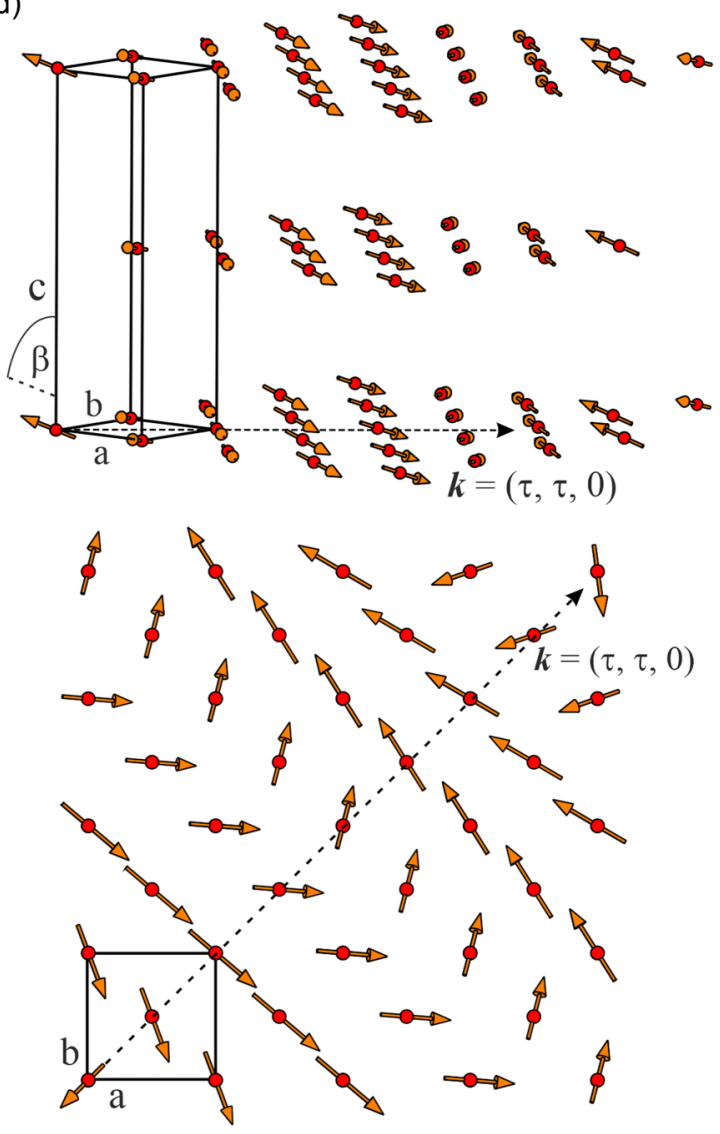

FIG. 3. Powder neutron diffraction study on $\mathrm{Sr}_{2} \mathrm{FeO}_{4}$. (a) Magnetic Bragg reflections at 0 and $5.5 \mathrm{~T}$ (red and green) obtained by subtraction of the pattern at $80 \mathrm{~K}$ from the ones at $1.8 \mathrm{~K}$. The intensity change of magnetic intensities between 0 and $5.5 \mathrm{~T}$ (black) is shown at the bottom. The magnetic satellites $(h k l)^{ \pm}$are generated with the propagation vector $\boldsymbol{k}=(0.137,0.137,0)$. (b) Temperature dependence of magnetic moments $\boldsymbol{\mu}_{\min }$ and $\boldsymbol{\mu}_{\text {maj }}$ and the vector component $k_{x}=\tau$. (c) Field dependence of the $(002)^{ \pm}$satellite intensity compared with the magnetization obtained from PPMS measurements. (d) Illustration of the magnetic structure of $\mathrm{Sr}_{2} \mathrm{FeO}_{4}$ in zero field. The magnetic moments of the $\mathrm{Fe}$ atoms form ferromagnetic layers perpendicular to the propagation vector $\boldsymbol{k}$ pointing along the [110] direction. At the bottom the magnetic structure is projected onto the $a b$ plane. The moments are tilted away from the $c$ axis by an angle $\beta \sim 70^{\circ}$.

transition, we use Model I (Table I) at zero field with spin ellipses in the crystallographic $a b$ plane. A vertical field disturbs the $4 \pi$ symmetry of the spatial distribution of ellipses for powder specimens and a $2 \pi$ cylindrical symmetry around the field axis becomes probable. The standard Rietveld method is not directly applicable to analyze the diffraction pattern since one must include a preferred orientation of spin ellipses "decoupled" from the $4 \pi$ symmetric distribution of the powder grains. To discuss the transition, we need a model to describe the field-dependent orientation of the ellipses during the spin-flop process. At zero field the ellipses, which are randomly oriented in $4 \pi$, intersect the SF plane. Those spins of the ellipses pointing along the intersection line already lie inside the SF plane. A plausible model for the reorientation of ellipses induced by the field will be a flop into the horizontal SF plane around this intersection line. Thereafter, ellipses are randomly distributed inside the plane. The intensity measured by a diffractometer defines a scattering vector $\mathbf{q}$ which selects just one direction inside the horizontal plane. Only grains with an orientation satisfying the diffraction condition must be considered. To account for this we label the analyzed two reflections $(\tau \tau 0)_{\mathbf{q}}$ and $(\tau \tau 2)_{\mathbf{q}}$ by a subscript $\mathbf{q}$. The fieldinduced magnetization generating only very weak intensity on nuclear reflections will not be considered.

First we discuss the $(\tau \tau 0)_{\mathbf{q}}$ reflection. The cycloidal spiral at zero field is described by an ellipse with $\boldsymbol{\mu}_{\min }$ along $(\tau, \tau$, $0)$. The latter is already the intersection line, and induced by an applied field, the spin ellipses flop around $\boldsymbol{\mu}_{\min }$. The diffraction condition constrains measured intensity to the scattering vector $(\tau, \tau, 0)_{\mathbf{q}}$ and thus the "flop axis" $\boldsymbol{\mu}_{\min }$ points along $(\tau, \tau, 0)_{\mathbf{q}}$ as well. Hence, $\boldsymbol{\mu}_{\min }$ remains along the scattering vector during the flop transition and magnetic scattering by $\boldsymbol{\mu}_{\text {min }}$ is forbidden. Measured magnetic scattering arises solely from the perpendicular component $\boldsymbol{\mu}_{\text {maj }}$. If the absolute value of $\boldsymbol{\mu}_{\text {maj }}$ does not change during the flop the intensity for the $(\tau \tau 0)_{\mathbf{q}}$ reflection will not change either. The above introduced flop model is in agreement with the experimental observation of a field-independent intensity (Fig. 2). 
TABLE I. Results of neutron diffraction study of $\mathrm{Sr}_{2} \mathrm{FeO}_{4}$ carried out at $1.8 \mathrm{~K}$ in zero field as obtained from refinements using the integrated intensities (given as $F^{2}$ ) of the magnetic peaks for three different models (upper part). In Model II the spin ellipse turns around the axis along [1-10] and in Model III along [110]. If a circular helix is assumed for Model II (labeled as Model IIc) one obtains a poor fit between observed and calculated intensities resulting in an enlarged residual $R_{\mathrm{M}}$. The observed and calculated $F^{2}$ values of the satellites obtained for the different models are compared in the lower part of the table.

\begin{tabular}{|c|c|c|c|c|c|c|}
\hline & & Spin axis & Model I & $\begin{array}{l}\text { Model II } \\
\text { Elliptical }\end{array}$ & $\begin{array}{l}\text { Model IIc } \\
\text { Circular }\end{array}$ & $\begin{array}{l}\text { Model III } \\
\text { Elliptical }\end{array}$ \\
\hline$\mu_{\min }\left[\mu_{\mathrm{B}}\right]$ & & - & $1.9(2)$ & $2.0(3)$ & $2.8(1)$ & $1.9(2)$ \\
\hline$\mu_{a b}\left[\mu_{\mathrm{B}}\right]$ & & [110] & $1.9(2)$ & $1.8(3)$ & $2.1(1)$ & $1.9(2)$ \\
\hline$\mu_{c}\left[\mu_{\mathrm{B}}\right]$ & & [001] & 0 & $0.9(5)$ & $1.9(2)$ & 0 \\
\hline$\mu_{\text {maj }}\left[\mu_{\mathrm{B}}\right]$ & & - & $3.46(5)$ & $3.36(17)$ & $2.8(1)$ & $3.46(5)$ \\
\hline$\mu_{a b}\left[\mu_{\mathrm{B}}\right]$ & & [1-10] & $3.46(5)$ & $3.36(17)$ & $2.8(1)$ & $3.35(17)$ \\
\hline$\mu_{c}\left[\mu_{\mathrm{B}}\right]$ & & [001] & 0 & 0 & - & $0.9(5)$ \\
\hline$\mu_{\mathrm{min}} / \mu_{\mathrm{maj}}$ & & - & 0.55 & 0.61 & 1.00 & 0.56 \\
\hline$R_{\mathrm{M}}$ & & - & 0.050 & 0.055 & 0.094 & 0.054 \\
\hline$Q\left[\AA^{-1}\right]$ & $(h k l)_{\mathrm{M}}$ & $F_{\mathrm{M}, \mathrm{obs}}{ }^{2}$ & $F_{\mathrm{M}, \mathrm{cal}^{2}}{ }^{2}$ & $F_{\mathrm{M}, \mathrm{cal}^{2}}{ }^{2}$ & $F_{\mathrm{M}, \mathrm{cal}^{2}}$ & $F_{\mathrm{M}, \mathrm{cal}^{2}}$ \\
\hline 0.315 & $000^{ \pm}$ & 31.8 & 31.3 & 31.8 & 30.9 & 31.2 \\
\hline 1.064 & $002^{ \pm}$ & 37.0 & 35.0 & 34.7 & 29.1 & 36.8 \\
\hline 1.512 & $101^{-}$ & 17.1 & 17.0 & 17.1 & 20.5 & 16.4 \\
\hline 1.934 & $101^{+}$ & 16.7 & 17.6 & 17.7 & 19.3 & 17.2 \\
\hline 1.989 & $110^{-}$ & 15.8 & 21.5 & 21.8 & 21.2 & 21.4 \\
\hline 2.056 & $004^{ \pm}$ & 30.6 & 26.3 & 25.5 & 20.6 & 27.7 \\
\hline 2.086 & $103^{-}$ & 16.3 & 19.7 & 19.6 & 19.0 & 20.1 \\
\hline 2.409 & $103^{+}$ & 15.8 & 17.4 & 17.4 & 16.8 & 17.6 \\
\hline
\end{tabular}

The situation becomes different for the $(\tau \tau 2)_{\mathbf{q}}$ reflection. At zero field the scattering vector $(\tau, \tau, 2)$ is nearly perpendicular to the ellipses with $\boldsymbol{\mu}_{\text {min }}$ and $\boldsymbol{\mu}_{\text {maj }}$ inside the crystal $a b$ plane for the grains. This maximizes the intensity. In the SF phase the ellipses are reoriented and lie in the horizontal SF plane. Since the scattering vector $(\tau, \tau, 2)_{\mathbf{q}}$ is inside the SF plane as well, the intensity must become lower. Experimentally one finds half of the intensity at $5.5 \mathrm{~T}$ as shown in Fig. 3(c).

Summarizing, the SF model can describe the spin flop with the result that there will be no change in the size of $\mu_{\text {maj }}$ and perhaps $\boldsymbol{\mu}_{\text {min }}$. The available data do not allow to obtain more quantitative results. The analysis indicates that the cycloidal type of the spin spiral is not affected by fields below $6.5 \mathrm{~T}$. This together with the weak temperature dependence of the wave vector [Fig. 3(b)] shows that the magnetic structure is quite robust.

The observation of a SF transition near $5 \mathrm{~T}$ in $\mathrm{Sr}_{2} \mathrm{FeO}_{4}$ suggests a moderate anisotropy field $H_{\mathrm{A}}$ which, together with the competing exchange interactions, is expected to determine the magnetic phase diagram, similar as for helical magnets [45].

\section{Synchrotron powder $x$-ray diffraction}

The insulating ground state of several $\mathrm{Fe}^{4+}$ oxides is stabilized by a $\mathrm{CD}$ of $\mathrm{Fe}^{4+}$, contrary to $\mathrm{Sr}_{2} \mathrm{FeO}_{4}$ where the Mössbauer spectra confirm the absence of a $\mathrm{CD}$. Previous Raman data showed an oxygen phonon mode even in the paramagnetic phase which is not intrinsic to the $\mathrm{K}_{2} \mathrm{NiF}_{4}$-type crystal structure [33] and which was confirmed in the present study [Fig. 4(a)]. Most remarkably, the elliptical cycloidal spin spiral also appears to be incompatible with $I 4 / \mathrm{mmm}$ symmetry [46]. To search for subtle structural distortions we have collected synchrotron powder x-ray diffraction patterns at beamline P02.1 of PETRA III, DESY, Hamburg, at temperatures of 100, 300, and $500 \mathrm{~K}$ but failed to detect clear signs of symmetry lowering [Fig. 4(b) and Ref. [43]]. All patterns could be reasonably well refined in space group $I 4 / \mathrm{mmm}$. In this space group the $\mathrm{Sr}$ and $\mathrm{O} 1$ atoms are located at the Wyckoff position $4 e(0,0, z)$, while the atoms $\mathrm{Fe}$ and $\mathrm{O} 2$ are located at the positions $2 a(0,0,0)$ and $4 c(0,1 / 2,0)$, respectively. The refinements included the overall scale factor, two positional and three isotropic thermal parameters. The experimental and calculated patterns at 100 and $500 \mathrm{~K}$ are shown in Fig. 4(b), the refined structural parameters of all data and calculated Fe-O bond lengths are listed in Table S1.

It is noted that no superstructure reflections were found in any of the X-ray and powder neutron patterns. Therefore, it can be assumed that the $I$ centering is not lost. A closer inspection of the powder patterns revealed that some specific reflections are slightly broadened. A peak-shape analysis (Fig. S2) revealed a systematic peak broadening for $h k l$ 's with higher $l$ contribution. Further it is found that the $00 l$ reflections show a pure Lorentzian peak shape, while $h k 0$ reflections show an increased Gaussian contribution. This behavior may be ascribed to the presence of stacking faults along the [001] direction. Therefore, we could not verify symmetry lowering from the synchrotron powder patterns.

A candidate for a lower-symmetric $I$-centered tetragonal space group could be the acentric and polar space group $14 \mathrm{~mm}$ (No. 107). In this setting both the $\mathrm{Sr}$ and $\mathrm{O} 1$ atoms split into the positions $2 a(0,0, z)(\mathrm{Sr} 1, \mathrm{O} 11)$ and $2 a\left(0,0,-z^{\prime}\right)(\mathrm{Sr} 2$, $\mathrm{O} 12)$. The second $\mathrm{O} 2$ atom is located at $4 b(0,1 / 2, z)$, and the Fe atom also at $2 a(0,0, z)$. In $I 4 m m$ the Fe atom could slightly rise up out of the horizontal $\mathrm{O} 2{ }_{4}$ plane. However, due 
(a)

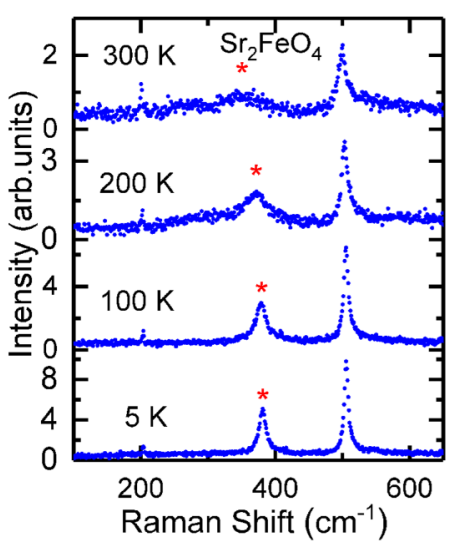

(b)

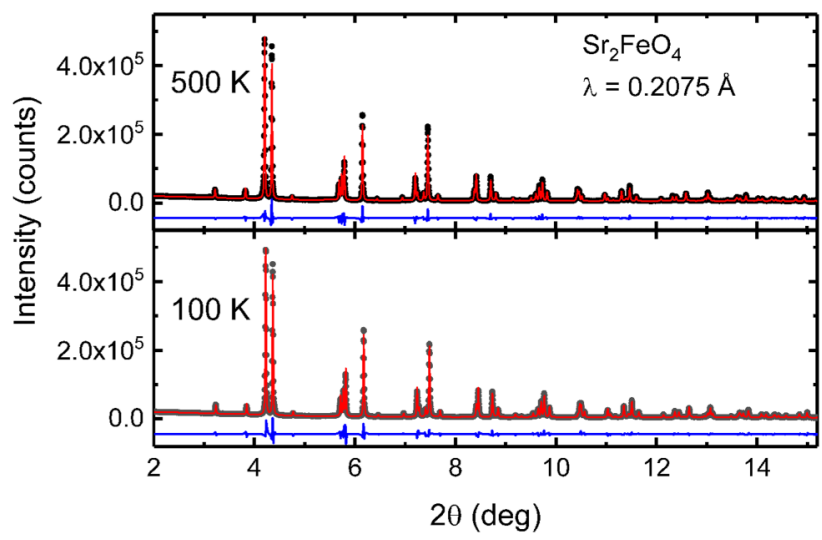

FIG. 4. (a) Raman spectra of $\mathrm{Sr}_{2} \mathrm{FeO}_{4}$ at the indicated temperatures. The star marks an anomalous Raman band referred to in the text. (b) Synchrotron powder x-ray diffraction patterns measured at 100 and $500 \mathrm{~K}$. The calculated patterns for space group $I 4 / \mathrm{mmm}$ (red solid lines) are compared with the observations (black-filled circles). The difference patterns $\left(I_{\mathrm{obs}}-I_{\mathrm{cal}}\right)$ (blue solid lines) are also shown.

to strongly correlated parameters this space group could not be proven from the synchrotron powder patterns. As space group $I 4 \mathrm{~mm}$ would allow for ferroelectricity it is desirable to study the electrical polarization properties of $\mathrm{Sr}_{2} \mathrm{FeO}_{4}$. On the other hand, the cycloidal elliptical spin structure suggests further symmetry lowering to at least orthorhombic symmetry [46].

\section{E. Mössbauer spectra at ambient pressure}

An elliptical spin spiral leads to a modulation of the magnetic moments and in turn to a distribution of hyperfine fields $B_{\mathrm{hf}}$ [47], which provides an explanation for the complex Mössbauer spectra of $\mathrm{Sr}_{2} \mathrm{FeO}_{4}$ below $T_{\mathrm{N}}$ [Fig. 5(a) and Refs. [16,32]]. Above $T_{\mathrm{N}}$ the spectra consist of a single quadrupole doublet. To reproduce the essential features of the spectrum at $6 \mathrm{~K}$ we have fitted it by assuming a superposition of eight six-line patterns where each component had the same isomer shift (IS $=0.073(1) \mathrm{mm} / \mathrm{s}$ ), and the same linewidth $(\Gamma=0.277(3) \mathrm{mm} / \mathrm{s})$. The relative intensities were allowed to vary. The resulting $B_{\mathrm{hf}}$ values and the corresponding values of the quadrupole shift $2 \varepsilon$ are shown in Fig. 5(b). It is evident that the $B_{\mathrm{hf}}$ and $2 \varepsilon$ values of the components are correlated with each other. In fact, assuming a linear correlation between $B_{\mathrm{hf}}$ and $2 \varepsilon$ the main shape of the spectrum can be reproduced if the $B_{\mathrm{hf}}$ distribution is extracted according to the HesseRübartsch method implemented in the MOSSWINN program [42] [Figs. 5(c) and 5(d)].

In an elliptical spin spiral the moment $\mu$ changes on rotation from a maximal value $\mu_{\text {maj }}$ (long axis) to a minimal value $\mu_{\min }$ (short axis) and as $B_{\mathrm{hf}}$ is essentially expected to be proportional to $\mu$ for the $\mathrm{Fe}^{4+} t_{2 g}{ }^{3} e_{g}{ }^{1}$ electron configuration having no first-order angular momentum, also $B_{\mathrm{hf}}$ shows a similar modulation. For a planar noncollinear elliptical spin structure and an incommensurate propagation vector the distribution $P_{\text {ell }}\left(B_{\mathrm{hf}}\right)$ of hyperfine fields is given by the relation [47]

$$
P_{\mathrm{ell}}\left(B_{\mathrm{hf}}\right) \propto \frac{h}{\sqrt{\left(h^{2}-y^{2}\right)\left(1-h^{2}\right)}},
$$

where $h=B_{\mathrm{hf}} / B_{\mathrm{hf}}^{\max }$ and $y=B_{\mathrm{hf}}^{\min } / B_{\mathrm{hf}}^{\max }$, and $B_{\mathrm{hf}}^{\max }$ and $B_{\mathrm{hf}}{ }^{\min }$ correspond to the maximal and minimal $B_{\mathrm{hf}}$ values $\left(B_{\mathrm{hf}}^{\min } \leqslant B_{\mathrm{hf}} \leqslant B_{\mathrm{hf}}^{\max }\right)$, respectively. If we use $B_{\mathrm{hf}}{ }^{\min }=24.2 \mathrm{~T}$ and $B_{\mathrm{hf}}{ }^{\max }=33.6 \mathrm{~T}$ obtained from the data evaluation with eight sextets as estimates we can calculate the $B_{\mathrm{hf}}$ distribution expected for an incommensurate elliptical spin structure according to Eq. (1) [Fig. 5(e)]. The experimental $B_{\mathrm{hf}}$ distribution in Fig. 5(d) reflects the main features of the calculated distribution and thus supports the scenario of an elliptical spin spiral. The ratio $B_{\mathrm{hf}}{ }^{\min } / B_{\mathrm{hf}}{ }^{\max }=0.72$ is somewhat larger than the moment ratios $\mu_{\min } / \mu_{\text {maj }}=0.56$ or 0.61 obtained from neutron powder data. The difference may reflect anisotropic contributions to $B_{\mathrm{hf}}$ [16]. Anisotropic dipolar and orbital contributions to $B_{\mathrm{hf}}$, however, cannot account for the large variation in $B_{\mathrm{hf}}$ owing to the lack of a first-order angular momentum and a quite small quadrupole splitting.

Note that Eq. (1) also holds for a collinear sinusoidally modulated spin structure. In this case the moments and thus $B_{\mathrm{hf}}{ }^{\mathrm{min}}$ can become zero. Thus, $y=0$ and

$$
P_{\mathrm{sin}}\left(B_{\mathrm{hf}}\right) \propto \frac{1}{\sqrt{\left(1-h^{2}\right)}}
$$

In this case the $B_{\mathrm{hf}}$ distribution is much broader than for an elliptical spin structure and it extends to zero. This leads to a pronounced central component in the Mössbauer spectra near zero velocity which is clearly not seen in the experimental spectrum at $6 \mathrm{~K}$. Thus, a sinusoidally modulated spin structure is not compatible with the Mössbauer spectra.

The Mössbauer spectra show that not only $B_{\mathrm{hf}}$ but also that the quadrupole splitting parameter $2 \varepsilon=e Q V_{Z Z} / 4\left(3 \cos ^{2} \theta-1\right)$ is modulated. Here, $V_{\mathrm{ZZ}}$ is the principal axis of the axially symmetric electric-field-gradient tensor, $\mathrm{Q}$ the nuclear quadrupole moment, and $\theta$ the angle between $V_{\mathrm{ZZ}}$ and $B_{\mathrm{hf}}$. If the spins and accordingly $B_{\mathrm{hf}}$ would lie completely in the $a b$ plane no modulation of $\theta$ and thus of $2 \varepsilon$ would be expected as for tetragonal $\mathrm{Sr}_{2} \mathrm{FeO}_{4} V_{\mathrm{ZZ}}$ should be parallel to the $c$ direction and thus $\theta=90^{\circ}$, independent of the spin orientation in the $a b$ plane. This suggests that the spin ellipses may be tilted somewhat out of the tetragonal plane as discussed above. A refined modeling of the Mössbauer spectra including the 
(a)

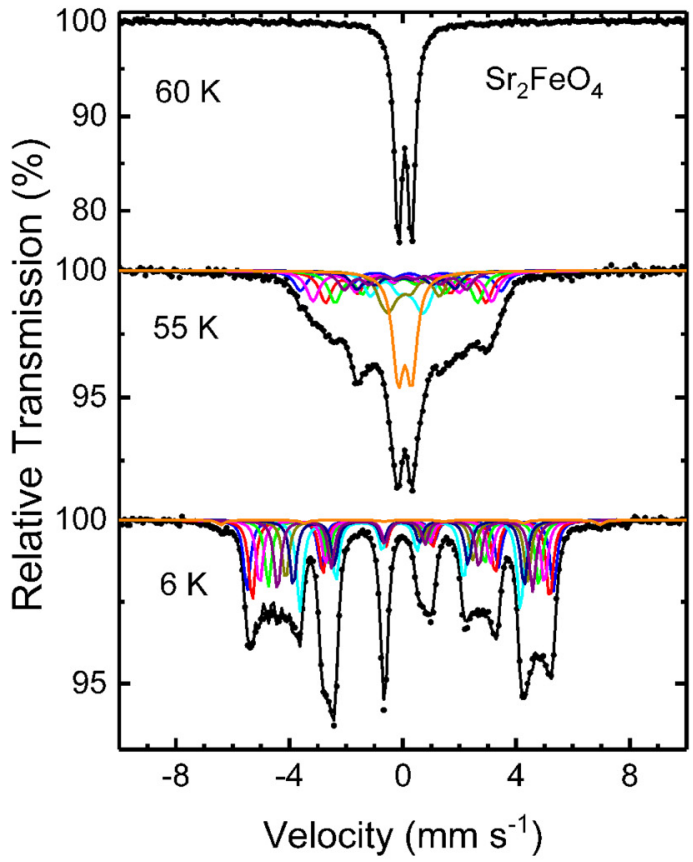

(b)

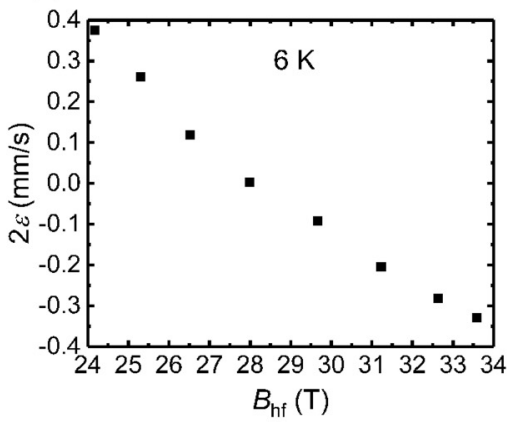

(d)

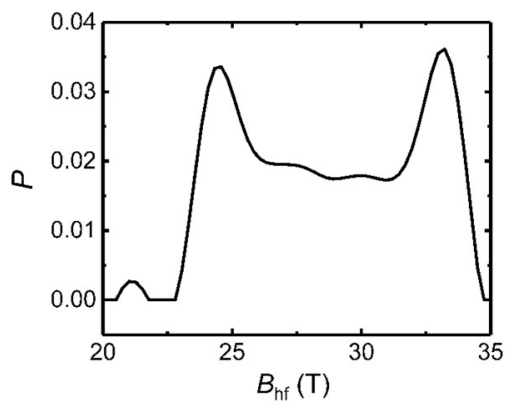

(c)

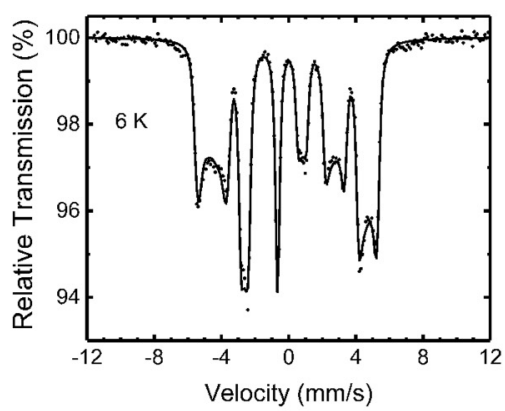

(e)

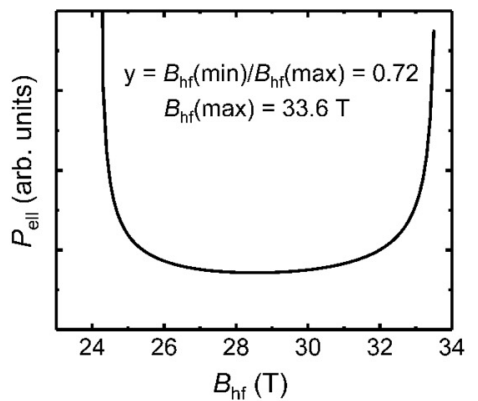

FIG. 5. ${ }^{57} \mathrm{Fe}$ Mössbauer spectra of $\mathrm{Sr}_{2} \mathrm{FeO}_{4}$ (a) Mössbauer spectra at the indicated temperatures. The sample was enriched with ${ }^{57} \mathrm{Fe}$ to $20 \%$. Dots correspond to experimental data, solid lines to calculated spectra. The spectrum at $6 \mathrm{~K}$ was fitted by using a superposition of eight hyperfine components (colored lines). (b) Hyperfine fields $B_{\mathrm{hf}}$ and corresponding quadrupole shifts $2 \varepsilon$ obtained from this fit model. (c) Fit of the spectrum at $6 \mathrm{~K}$ obtained by assuming a linear correlation between $B_{\mathrm{hf}}$ and $2 \varepsilon$ and extracting a $B_{\mathrm{hf}}$ distribution which is shown in (d). (e) $B_{\mathrm{hf}}$ distribution calculated for an incommensurate elliptical spin structure according to Eq. (1).

anisotropy of $B_{\mathrm{hf}}$ may allow further clues about the detailed orientation of the spin ellipses [48].

\section{F. Change of spin structure at high pressure}

It was shown previously that the unusual Raman band vanishes at pressures above $6 \mathrm{GPa}$ [33] and Mössbauer spectra revealed an anomaly of the quadrupole splitting in the same pressure range [34]. Furthermore, metallization near $P_{\mathrm{m}}=18 \mathrm{GPa}$ and a simple six-line hyperfine pattern in the metallic regime were reported [34]. Here, we have measured ${ }^{57} \mathrm{Fe}$ Mössbauer spectra at pressures of 5 and $8 \mathrm{GPa}$ (Fig. 6) using the synchrotron Mössbauer source at the $\mathrm{Nu}$ clear Resonance beamline ID18 of the ESRF, Grenoble. While the 5-GPa spectrum still reveals a complex hyperfine pattern (average $B_{\mathrm{hf}}=29.9 \mathrm{~T}$ ), the 8 -GPa spectrum shows a simple six-line pattern the $B_{\mathrm{hf}}$ of which $(28.8 \mathrm{~T})$ is reduced by $1.4 \mathrm{~T}$ in an external longitudinal field of $2 \mathrm{~T}$. This shift indicates a ferromagnetic spin alignment, although the field of $2 \mathrm{~T}$ is not sufficient to completely align the ferromagnetic domains. The Mössbauer spectra demonstrate that the elliptical spin spiral is transformed to a ferromagnetic spin structure well below $P_{\mathrm{m}}$, just in the pressure range where the unusual Raman band disappears. This supports the scenario of a subtle structural distortion which is suppressed near $6 \mathrm{GPa}$ due to increasing itineracy of the electronic system. In fact, the resistance shows a drop in this pressure range although a small gap persists up to $18 \mathrm{GPa}$ [34]. The change in spin structure can be attributed

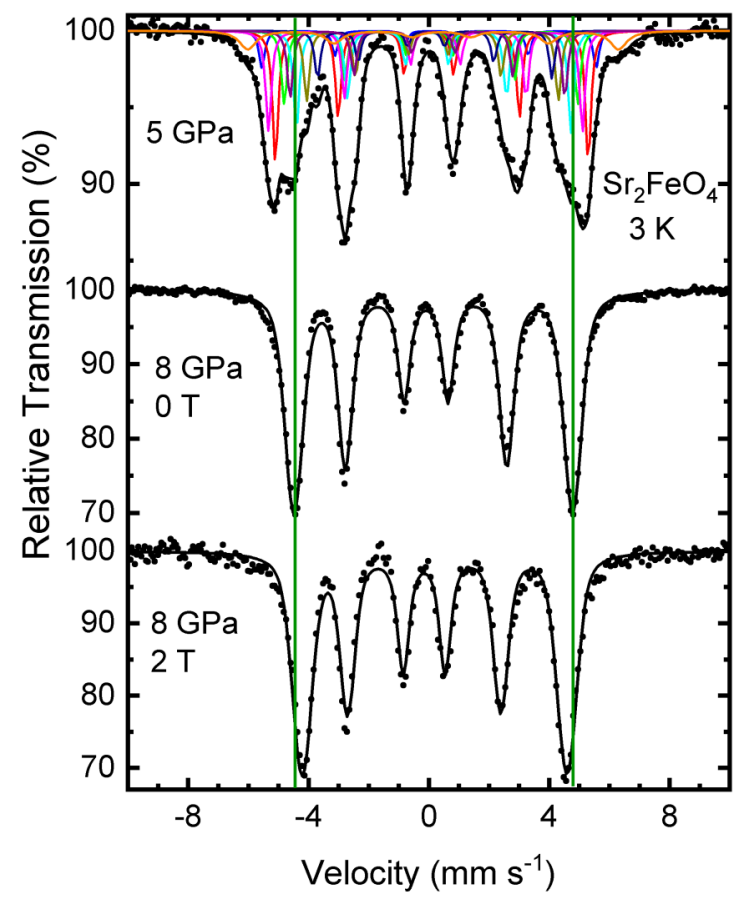

FIG. 6. Synchrotron Mössbauer spectra of $\mathrm{Sr}_{2} \mathrm{FeO}_{4}$ at 5 and $8 \mathrm{GPa}$ in zero field and at $8 \mathrm{GPa}$ in a longitudinal field of $2 \mathrm{~T}$. The vertical lines (green) highlight the outer line positions at $8 \mathrm{GPa}$ and 0 T. The samples used here were enriched with ${ }^{57} \mathrm{Fe}$ to $20 \%$ (8-GPa spectra) or $10 \%$ (5-GPa spectrum). 
to a strengthening of ferromagnetic NN-double exchange with respect to antiferromagnetic NNN interactions under pressure, which emphasizes the strong coupling among charge, spin, and lattice degrees of freedom in $\mathrm{Sr}_{2} \mathrm{FeO}_{4}$.

\section{CONCLUSIONS}

We have shown that the negative charge-transfer gap insulator $\mathrm{Sr}_{2} \mathrm{FeO}_{4}$ adopts an elliptical cycloidal spin spiral below $T_{\mathrm{N}}=56 \mathrm{~K}$ which responds at moderate values of external parameters like magnetic field or pressure. Tuning of spiral spin structures is of importance for exploring topological spin textures in this class of compounds as well as for multiferroic systems [49,50]. In fact, it may also be instructive to study the electrical polarization properties of $\mathrm{Sr}_{2} \mathrm{FeO}_{4}$. The insulating ground state of $\mathrm{Sr}_{2} \mathrm{FeO}_{4}$ appears to be stabilized by a hidden structural distortion which, similar to the checkerboard charge order in the related $\mathrm{Sr}_{3} \mathrm{Fe}_{2} \mathrm{O}_{7}$ [51], is invisible to standard diffraction techniques and can be considered as an alternative to the $\mathrm{CD}$ in $\mathrm{Fe}^{4+}$ and other oxides. For elucidating the role of anisotropy it would be desirable to study single crystals of $\mathrm{Sr}_{2} \mathrm{FeO}_{4}$. Unfortunately, $\mathrm{Sr}_{2} \mathrm{FeO}_{4}$ decomposes above $700{ }^{\circ} \mathrm{C}$ which excludes many convenient techniques for the growth of single crystals of transition metal oxides. However, it may be possible to stabilize thin films of $\mathrm{Sr}_{2} \mathrm{FeO}_{4}$ by proper choice of substrate and deposition conditions, similar as in case of $\mathrm{SrFeO}_{3}[52]$.

\section{ACKNOWLEDGMENTS}

We thank G. Takur for support with Rietveld refinements of XRD data. We gratefully acknowledge the ESRF for granting beamtime at the Nuclear Resonance beamline ID18 for experiment HC-4292. We thank J.-P. Celse for technical assistance during the beamtime at ID18. We acknowledge DESY (Hamburg, Germany), a member of the Helmholtz Association HGF, for the provision of experimental facilities. Parts of this research were carried out at PETRA III beamline P02.1. D.C.P. was supported by the Deutsche Forschungsgemeinschaft (DFG) through project $\mathrm{C} 03$ of the Collaborative Research Center SFB 1143 (Project id No. 247310070); Project id No. 455319354; and the Würzburg-Dresden Cluster of Excellence on Complexity and Topology in Quantum Matter - ct.qmat (EXC 2147, Project id No. 390858490).
[1] E. Dagotto, Complexity in strongly correlated electronic systems, Science 309, 257 (2005).

[2] P. Dai, J. Hu, and E. Dagotto, Magnetism and its microscopic origin in iron-based high-temperature superconductors, Nat. Phys. 8, 709 (2012).

[3] J. Zaanen, G. A. Sawatzky, and J. W. Allen, Band Gaps and Electronic Structure of Transition-Metal Compounds, Phys. Rev. Lett. 55, 418 (1985).

[4] T. Mizokawa, H. Namatame, A. Fujimori, K. Akeyama, H. Kondoh, H. Kuroda, and N. Kosugi, Origin of the Band Gap in the Negative Charge-Transfer-Energy Compound $\mathrm{NaCuO}_{2}$, Phys. Rev. Lett. 67, 1638 (1991).

[5] J. A. Alonso, J. L. García-Muñoz, M. T. Fernández-Díaz, M. A. G. Aranda, M. J. Martínez-Lope, and M. T. Casais, Charge Disproportionation in $\mathrm{RNiO}_{3}$ Perovskites: Simultaneous MetalInsulator and Structural Transition in $\mathrm{YNiO}_{3}$, Phys. Rev. Lett. 82, 3871 (1999).

[6] T. Mizokawa, D. I. Khomskii, and G. A. Sawatzky, Spin and charge ordering in self-doped Mott insulators, Phys. Rev. B. 61, 11263 (2000).

[7] H. Park, A. J. Millis, and C. A. Marianetti, Site-Selective Mott Transition in Rare-Earth-Element Nickelates, Phys. Rev. Lett. 109, 156402 (2012).

[8] S. Johnston, A. Mukherjee, I. Elfimov, M. Berciu, and G. A. Sawatzky, Charge Disproportionation without Charge Transfer in the Rare-Earth-Element Nickelates as a Possible Mechanism for the Metal-Insulator Transition, Phys. Rev. Lett. 112, 106404 (2014).

[9] R. J. Green, M. W. Haverkort, and G. A. Sawatzky, Bond disproportionation and dynamical charge fluctuations in the perovskite rare-earth nickelates, Phys. Rev. B 94, 195127 (2016).

[10] I. I. Mazin, D. I. Khomskii, R. Lengsdorf, J. A. Alonso, W. G. Marshall, R. M. Ibberson, A. Podlesnyak, M. J. Martinez-Lope, and M M., Abd-Elmeguid, Charge Ordering as Alternative to Jahn-Teller Distortion, Phys. Rev. Lett. 98, 176406 (2007).
[11] V. Bisogni, S. Catalano, R. J. Green, M. Gibert, R. Scherwitzl, Y. Huang, V. N. Strocov, P. Zubko, S. Balandeh, J.-M. Tricone, G. Sawatzky, and T. Schmitt, Ground-state oxygen holes and the metal-insulator transition in the negative charge-transfer rare-earth nickelates, Nat. Commun. 7, 13017 (2016).

[12] E. Wawrzyńska, R. Coldea, E. M. Wheeler, I. I. Mazin, M. D. Johannes, T. Sörgel, M. Jansen, R. M. Ibberson, and P. G. Radaelli, Orbital Degeneracy Removed by Charge Order in Triangular Antiferromagnet $\mathrm{AgNiO}_{2}$, Phys. Rev. Lett. 99, 157204 (2007)

[13] E. Wawrzyńska, R. Coldea, E. M. Wheeler, T. Sörgel, M. Jansen, R. M. Ibberson, P. G. Radaelli, and M. M. Koza, Charge disproportionation and collinear magnetic order in the frustrated triangular antiferromagnet $\mathrm{AgNiO}_{2}$, Phys. Rev. B 77, 094439 (2008).

[14] M. Takano, N. Nakanishi, Y. Takeda, S. Naka, and T. Takada, Charge disproportionation in $\mathrm{CaFeO}_{3}$ studied with the Mössbauer effect, Mater. Res. Bull. 12, 923 (1977).

[15] P. M. Woodward, D. E. Cox, E. Moshopoulou, A. W. Sleight, and S. Morimoto, Structural studies of charge disproportionation and magnetic order in $\mathrm{CaFeO}_{3}$, Phys. Rev. B 62, 844 (2000).

[16] S. E. Dann, M. T. Weller, D. B. Currie, M. F. Thomas, and A. D. Al-Rawwas, Structure and magnetic properties of $\mathrm{Sr}_{2} \mathrm{FeO}_{4}$ and $\mathrm{Sr}_{3} \mathrm{Fe}_{2} \mathrm{O}_{7}$ studied by powder neutron diffraction and Mössbauer spectroscopy, J. Mater. Chem. 3, 1231 (1993).

[17] P. Adler, Electronic state, magnetism, and electrical transport behavior of $\mathrm{Sr}_{3} \mathrm{~A}_{x} \mathrm{Fe}_{2} \mathrm{O}_{7}(x \leqslant 0.4, A=\mathrm{Ba}, \mathrm{La})$, J. Solid State. Chem. 130, 129 (1997).

[18] P. Adler, U. Schwarz, K. Syassen, G. Kh. Rozenberg, G. Yu. Machiavariani, A. P. Milner, M. P. Pasternak, and M. Hanfland, Collapse of the charge disproportionation and covalency-driven insulator-metal transition in $\mathrm{Sr}_{3} \mathrm{Fe}_{2} \mathrm{O}_{7}$ under pressure, Phys. Rev. B 60, 4609 (1999). 
[19] K. Kuzushita, S. Morimoto, S. Nasu, and S. Nakamura, Charge disproportionation and antiferromagnetic order of $\mathrm{Sr}_{3} \mathrm{Fe}_{2} \mathrm{O}_{7}$, J. Phys. Soc. Jpn. 69, 2767 (2000).

[20] P. K. Gallagher, J. B. MacChesney, and D. N. E. Buchanan, Mössbauer effect in the system $\mathrm{SrFeO}_{2.5-3.0}$, J. Chem. Phys. 41, 2429 (1964).

[21] J. B. MacChesney, R. C. Sherwood, and J. F. Potter, Electrical and magnetic properties of the strontium ferrates, J. Chem. Phys. 43, 1907 (1965)

[22] N. Hayashi, T. Yamamoto, H. Kageyama, M. Nishi, Y. Watanabe, T. Kawakami, Y. Matsushita, A. Fujimori, and M. Takano, $\mathrm{BaFeO}_{3}$ : A ferromagnetic iron oxide, Angew. Chem., Int. Ed. 50, 12547 (2011).

[23] A. E. Bocquet, A. Fujimori, T. Mizokawa, T. Saitoh, H. Namatame, S. Suga, N. Kimizuka, Y. Takeda, and M. Takano, Electronic structure of $\mathrm{SrFe}^{4+} \mathrm{O}_{3}$ and related Fe perovskites, Phys. Rev. B 45, 1561 (1992).

[24] T. Takeda, Y. Yamaguchi, and H. Watanabe, Magnetic structure of $\mathrm{SrFeO}_{3}$, J. Phys. Soc. Jpn. 33, 967 (1972).

[25] J.-H. Kim, A. Jain, M. Reehuis, D. C. Peets, C. Ulrich, J. T. Park, E. Faulhaber, A. Hoser, H. C. Walker, D. T. Adroja, A. C. Walters, D. S. Inosov, A. Maljuk, and B. Keimer, Competing Exchange Interactions at the Verge of a Metal-Insulator Transition in the Two-Dimensional Spiral Magnet $\mathrm{Sr}_{3} \mathrm{Fe}_{2} \mathrm{O}_{7}$, Phys. Rev. Lett. 113, 147206 (2014).

[26] M. Mostovoy, Helicoidal Ordering in Iron Perovskites, Phys. Rev. Lett. 94, 137205 (2005).

[27] A. Lebon, P. Adler, C. Bernhard, A. V. Boris, A. V. Pimenov, A. Maljuk, C. T. Lin, C. Ulrich, and B. Keimer, Magnetism, Charge order, and Giant Magnetoresistance in $\mathrm{SrFeO}_{3-\delta}$ Single Crystals, Phys. Rev. Lett. 92, 037202 (2004).

[28] S. Ishiwata, M. Tokunaga, Y. Kaneko, D. Okuyama, S. Wakimoto, K. Kakurai, T. Arima, Y. Taguchi, and Y. Tokura, Versatile helimagnetic phases under magnetic fields in cubic perovskite $\mathrm{SrFeO}_{3}$, Phys. Rev. B 84, 054427 (2011).

[29] S. Chakraverty, T. Matsuda, H. Wadati, J. Okamoto, Y. Yamasaki, H. Nakao, Y. Murakami, S. Ishiwata, M. Kawasaki, Y. Taguchi, Y. Tokura, and H. Y. Hwang, Multiple helimagnetic phases and topological Hall effect in epitaxial thin films of pristine and Co-doped $\mathrm{SrFeO}_{3}$, Phys. Rev. B 88, 220405(R) (2013).

[30] S. Ishiwata, T. Nakajima, J. H. Kim, D. S. Inosov, N. Kanazawa, J. S. White, J. L. Gavilano, R. Georgii, K. Seemann, G. Brandl, P. Manuel, D. D. Khalyavin, S. Seki, Y. Tokunaga, M. Kinoshita, Y. W. Long, Y. Kaneko, Y. Taguchi, T. Arima, B. Keimer, and Y. Tokura, Emergent topological spin structures in the centrosymmetric cubic perovskite $\mathrm{SrFeO}_{3}$, Phys. Rev. B 101, 134406 (2020).

[31] S. E. Dann, M. T. Weller, and D. B. Currie, Synthesis and structure of $\mathrm{Sr}_{2} \mathrm{FeO}_{4}$, J. Solid State. Chem. 92, 237 (1991).

[32] P. Adler, Properties of $\mathrm{K}_{2} \mathrm{NiF}_{4}$-type Oxides $\mathrm{Sr}_{2} \mathrm{FeO} \sim 4$, J. Solid State. Chem. 108, 275 (1994).

[33] P. Adler, A. F. Goncharov, K. Syassen, and E. Schönherr, Optical reflectivity and Raman spectra of $\mathrm{Sr}_{2} \mathrm{FeO}_{4}$ under pressure, Phys. Rev. B 50, 11396 (1994).

[34] G. Kh. Rozenberg, A. P. Milner, M. P. Pasternak, G. R. Hearne, and R. D. Taylor, Experimental confirmation of a $p-p$ intraband gap in $\mathrm{Sr}_{2} \mathrm{FeO}_{4}$, Phys. Rev. B 58, 10283 (1998).

[35] C. Linke and M. Jansen, Über $\mathrm{Ag}_{2} \mathrm{SnO}_{3}$, das erste Silberstannat, Z. Anorg. Allg. Chem. 623, 1441 (1997).
[36] Helmholtz-Zentrum für Materialien und Energie, E2: The flatcone diffractometer at BER II, J. Large-Scale Res. Fac. 4, A129 (2018).

[37] N. Stüßer and N. M. Hofmann, An adjustable in-pile fan collimator for focusing at a neutron diffractometer, Nucl. Instrum. Methods Phys. Res. Sect. A 482, 744 (2002).

[38] J. Rodríguez-Carvajal, Recent advances in magnetic structure determination by neutron powder diffraction, Physica B. 192, 55 (1993).

[39] V. F. Sears, in International Tables for Crystallography, edited by A. J. C. Wilson (Kluwer, Dordrecht, 1995), Vol. C, p. 383; P. J. Brown, ibid., Vol. C, p. 391.

[40] V. Potapkin, A. I. Chumakov, G. V. Smirnov, J.-P. Celse, R. Rüffer, C. McCammon, and L. Dubrovinski, The ${ }^{57} \mathrm{Fe}$ synchrotron Mössbauer source at the ESRF, J. Synchrotron Rad. 19, 559 (2012).

[41] R. Rüffer and A. I. Chumakov, Nuclear-resonance beamline at ESRF, Hyperfine Interact. 97-98, 589 (1996).

[42] Z. Klencsár, E. Kuzmann, and A. Vértes, User-friendly software for Mössbauer spectrum analysis, J. Radioanal. Nucl. Chem. 210, 105 (1990).

[43] See Supplemental Material at http://link.aps.org/supplemental/ 10.1103/PhysRevB.105.054417 for additional magnetization data, structural data and line-shape analysis of synchrotron powder x-ray diffraction data.

[44] I. S. Jacobs, Spin-Flopping in $\mathrm{MnF}_{2}$ by high magnetic fields, J. Appl. Phys. 32, S61 (1961).

[45] D. C. Johnston, Magnetic structure and magnetization of $z$ axis helical Heisenberg antiferromagnets with XY anisotropy in high fields transverse to the helix axis at zero temperature, Phys. Rev. B 99, 214438 (2019).

[46] P. Fabrykiewicz, R. Przeniosło, and I. Sosnowska, Crystal symmetry for incommensurate helical and cycloidal modulations, Acta Cryst. A77, 160 (2021).

[47] D. Colson, A. Forget, and P. Bonville, The modulated antiferromagnetic structures in multiferroic $\mathrm{FeVO}_{4}$ : $\mathrm{A}^{57} \mathrm{Fe}$ Mössbauer spectroscopy investigation, J. Magn. Magn. Mater. 378, 529 (2015).

[48] A. V. Sobolev, A. A. Akulenko, I. S. Glazkova, D. A. Pankratov, and I. A. Presniakov, Modulated magnetic structure of $\mathrm{Fe}_{3} \mathrm{PO}_{7}$ as seen by ${ }^{57} \mathrm{Fe}$ Mössbuer spectroscopy, Phys. Rev. B 97, 104415 (2018).

[49] K. Taniguchi, N. Abe, H. Umetsu, H. Aruga Katori, and T. Arima, Control of the Magnetoelectric Domain-Wall Stability by a Magnetic Field in Multiferroic $\mathrm{MnWO}_{4}$, Phys. Rev. Lett. 101, 207205 (2008).

[50] I. Urcelay-Olabarria, E. Ressouche, A. A. Mukhin, V. Yu. Ivanov, A. M. Kadomtseva, Yu. F. Popov, G. P. Vorob'ev, A. M. Balbashov, J. L. Garcia-Muno, and V. Skumryev, X phase of $\mathrm{MnWO}_{4}$, Phys. Rev. B 90, 024408 (2014).

[51] J.-H. Kim, D. C. Peets, M. Reehuis, P. Adler, A. Maljuk, T. Ritschel, M. C. Allison, J. R. L. Mardegan, J. Geck, P. J. Bereciartua Perez, S. Francoual, A. C. Walters, T. Keller, P. M. Abdala, P. Pattison, P. Dosanjh, and B. Keimer, Hidden Charge Order in an Iron Oxide Square Lattice Compound, Phys. Rev. Lett. 127, 097203 (2021).

[52] N. Hayashi, T. Terashima, and M. Takano, Oxygen-holes creating different electronic phases in $\mathrm{Fe}^{4+}$-oxides: Successful growth of single crystalline films of $\mathrm{SrFeO}_{3}$ and related perovskites at low pressure, J. Mater. Chem. 11, 2235 (2001). 